\title{
Novel antibacterial compounds specifically targeting the essential WalR response regulator
}

\author{
Yasuhiro Gotoh ${ }^{1}$, Akihiro Doi ${ }^{1}$, Eiji Furuta ${ }^{1}$, Sarah Dubrac ${ }^{2}$, Yoshimasa Ishizaki ${ }^{3}$, Masato Okada ${ }^{4}$, \\ Masayuki Igarashi ${ }^{3}$, Norihiko Misawa ${ }^{5}$, Hirofumi Yoshikawa ${ }^{4}$, Toshihide Okajima ${ }^{6}$, Tarek Msadek ${ }^{2}$ \\ and Ryutaro Utsumi ${ }^{1}$
}

The WalK/WaIR (YycG/YycF) two-component system, which is essential for cell viability, is highly conserved and specific to low-GC percentage of Gram-positive bacteria, making it an attractive target for novel antimicrobial compounds. Recent work has shown that WaIK/WalR exerts an effect as a master regulatory system in controlling and coordinating cell wall metabolism with cell division in Bacillus subtilis and Staphylococcus aureus. In this paper, we develop a high-throughput screening system for WalR inhibitors and identify two novel inhibitors targeting the WalR response regulator (RR): walrycin A (4-methoxy-1naphthol) and walrycin B (1,6-dimethyl-3-[4-(trifluoromethyl)phenyl]pyrimido[5,4-e][1,2,4]triazine-5,7-dione). Addition of these compounds simultaneously affects the expression of WalR regulon genes, leading to phenotypes consistent with those of cells starved for the WalK/WalR system and having a bactericidal effect. $B$. subtilis cells form extremely long aseptate filaments and $S$. aureus cells form large aggregates under these conditions. These results show that walrycins $A$ and $B$ are the first antibacterial agents targeting WalR in $B$. subtilis and $S$. aureus.

The Journal of Antibiotics (2010) 63, 127-134; doi:10.1038/ja.2010.4; published online 29 January 2010

Keywords: antibacterial agent; inhibitor; two-component signal transduction system; response regulator; WalR (YycF)

\section{INTRODUCTION}

In bacteria, signal transduction in response to a wide variety of environmental stimuli is mediated by pairs of proteins that communicate with each other through a two-component signal transduction system (TCS, Figure 1) involving protein phosphorylation. TCS consists of a histidine kinase (HK) and a response regulator (RR) that have global roles in bacterial growth as well as in drug resistance, virulence, biofilm formation and quorum sensing. ${ }^{1}$ TCS inhibitors not only work as antibacterial agents but can also be developed as the inhibitors for drug resistance, biofilm formation, quorum sensing and virulence, as well as for plant-growth-regulating agents. In particular, TCSs are attractive as targets for antimicrobials for the following reasons. (1) Although many HK and RR genes are coded on the bacterial genome, few are found in lower eukaryotes. For example, only one HK and two RRs are present in the complete genome sequence of Saccharomyces cerevisiae, and none has been found from mammalian genomes. The HK/RR signal transduction system is distinct from serine/threonine and tyrosine phosphorylation in higher eukaryotes. (2) HKs and RRs possess a high degree of homology around their active sites. HKs are characterized by a conserved C-terminal catalytic domain containing a histidine residue, which is the site of autophosphorylation, and the N, G1, F and G2 boxes, which presumably form a nucleotide-binding surface. RRs contain a conserved $\mathrm{N}$-terminal domain that includes the aspartate residue that is phosphorylated, a pair of aspartate residues preceding this site and a lysine residue, all of which contribute to the acidic pocket for the phosphorylation site. Such a high degree of structural homology in the catalytic domain of HKs and in the receiver domain of RRs suggests that multiple TCSs within a single bacterium could be inhibited simultaneously by a single inhibitor, thereby lowering the frequency of the appearance of drug-resistant strains. (3) Some TCSs are essential for bacterial viability: YycG/YycF in Bacillus subtilis, Staphylococcus aureus, Streptococcus pneumoniae, Enterococcus faecalis, Streptococcus pyogenes, Streptococcus mutans and Streptococcus epidermidis. ${ }^{2,3}$ The YycG/YycF TCS, recently renamed WalK/WalR to reflect its function, ${ }^{2,4}$ is highly conserved and specific to low-G+C Grampositive bacteria, such as $B$. subtilis and $S$. aureus, in which it exerts an effect as a master regulatory system for cell wall metabolism and is essential for cell viability. ${ }^{4-8}$ As such, the WalK/WalR system constitutes an attractive target for the development of novel antimicrobial compounds, as inhibitors targeting WalK or WalR would be expected to have a bactericidal effect toward a broad range of major Grampositive pathogens. Previously, a number of HK inhibitors have been identified, ${ }^{9-12}$ but these studies did not conclusively show a direct

${ }^{1}$ Department of Bioscience, Graduate School of Agriculture, Kinki University, Nara, Japan; ${ }^{2}$ Institut Pasteur, Biology of Gram-Positive Pathogens, Paris, France; ${ }^{3}$ Microbial Chemistry Research Center, Shinagawa-ku, Tokyo, Japan; ${ }^{4}$ Department of Bioscience, Tokyo University of Agriculture, Tokyo, Japan; ${ }^{5}$ Central Laboratories for Frontier Technology, Kirin Holdings, Suematsu, Nonoichi-machi, Ishikawa, Japan and ${ }^{6}$ Institute of Scientific and Industrial Research, Osaka University, Ibaraki, Osaka, Japan Correspondence: Dr R Utsumi, Department of Bioscience, Graduate School of Agriculture, Kinki University, 3327-204 Nakamachi, Nara 631-8505, Japan. E-mail: utsumi@nara.kindai.ac.jp

Received 14 December 2009; revised and accepted 4 January 2010; published online 29 January 2010 


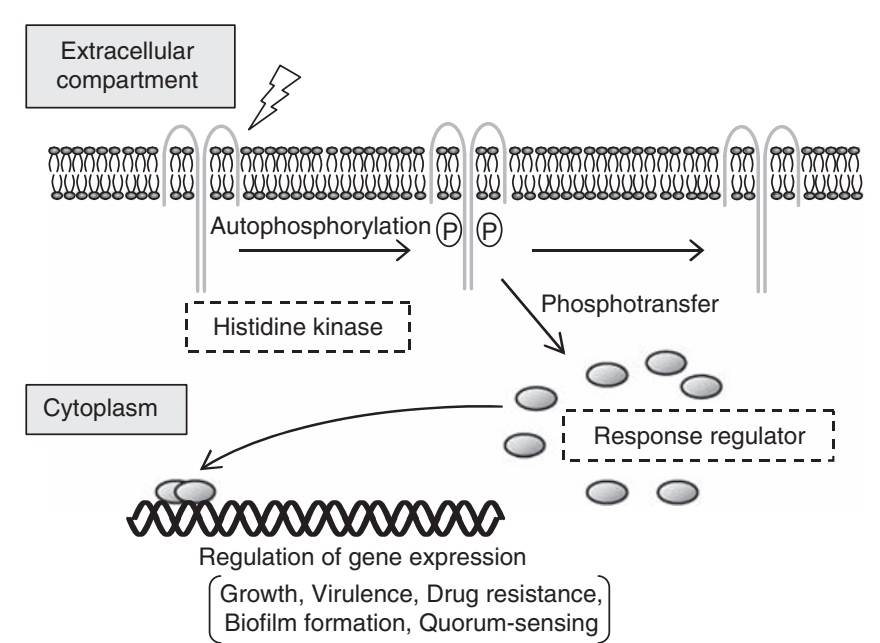

Figure 1 Two-component signal transduction system.

relationship between cell death and HK inhibition rather than some other uncharacterized nonspecific effect. However, structure-based virtual screening of compounds potentially targeting the kinase activity of $S$. epidermidis WalK found several to be active against not only S. epidermidis but also S. aureus, S. aureus and S. mutans. ${ }^{13}$

WalR is a typical RR of the OmpR/PhoB subfamily with a characteristic winged helix-turn-helix DNA-binding domain. ${ }^{14,15}$ These regulators control expression of their regulon genes by forming a head-to-head dimer of the receiver domains, using the conserved $\alpha 4-\beta 5-\alpha 5$ face for intermolecular interactions, paired with a headto-tail dimer of the winged helix-turn-helix motifs that bind to the tandem DNA repeats of their binding site. ${ }^{16}$ Inhibiting the cognate sensor kinase may not be sufficient to disrupt signaling, as RRs can often be phosphorylated by non-cognate sensor kinases ${ }^{17}$ or smallmolecule phosphate donors, ${ }^{18}$ making them the more attractive target for inhibition. ${ }^{16,19}$ Nevertheless, WalR-specific inhibitors have yet to be identified.

In this paper we describe a high-throughput screening system targeting the WalR RR, which uses a fusion between WalR and the DNA-binding domain of IclR to form a dimeric repressor and egfP as a reporter gene (Figure 2a). Using this system, we identified two novel inhibitors specifically targeting WalR in B. subtilis and S. aureus that were highly effective in killing these bacteria.

\section{MATERIALS AND METHODS}

\section{Bacteria strains and plasmids}

Bacterial strains and plasmids used in this study are listed in Table 1. Antibiotics were used at the following concentrations: ampicillin: $50 \mu \mathrm{g} \mathrm{ml}^{-1}$ for $B$. subtilis and $100 \mu \mathrm{g} \mathrm{m}^{-1}$ for E. coli; chloramphenicol: $5 \mu \mathrm{g} \mathrm{m}^{-1}$ for B. subtilis and $10 \mu \mathrm{g} \mathrm{ml}$ for S. aureus; kanamycin: $5 \mu \mathrm{g} \mathrm{ml}^{-1}$.

\section{Plasmid construction}

Primers used for plasmid construction are listed in Supplementary Table S1. To obtain pFI014, we PCR-amplified the DNA fragment containing the full length of walR of B. subtilis by using pBY33 as the template and YFIR4-399F and YFIR358-705R as the primers. The amplicons were digested with $K p n \mathrm{I}$ and ligated at the $K p n I$ site of pFI003 ${ }^{20}$ (Figure 2a). For pArcA and pOmpR, the DNA fragments were prepared by PCR using the genomic DNA from E. coli W3110 as the template and the following primers: pFI003-arcA-F and pFI003arcA-R for pArcA and pFI003-ompR-F and pFI003-ompR-R for pOmpR. The amplicons were digested with $K p n \mathrm{I}$ and ligated at the $K p n \mathrm{I}$ site of pFI003.

B. subtilis walR was also amplified by PCR with pBY33 as the template and primers pANBF- 1 and BsYycF-R. The PCR product was digested with KpnI and a

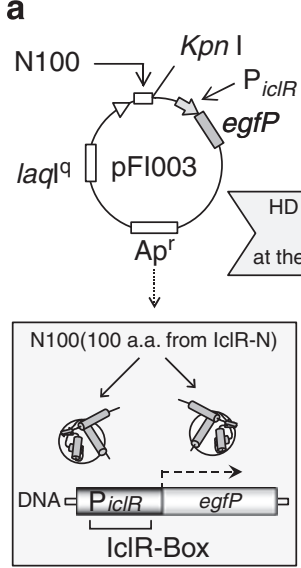

Expression of EGFP

b
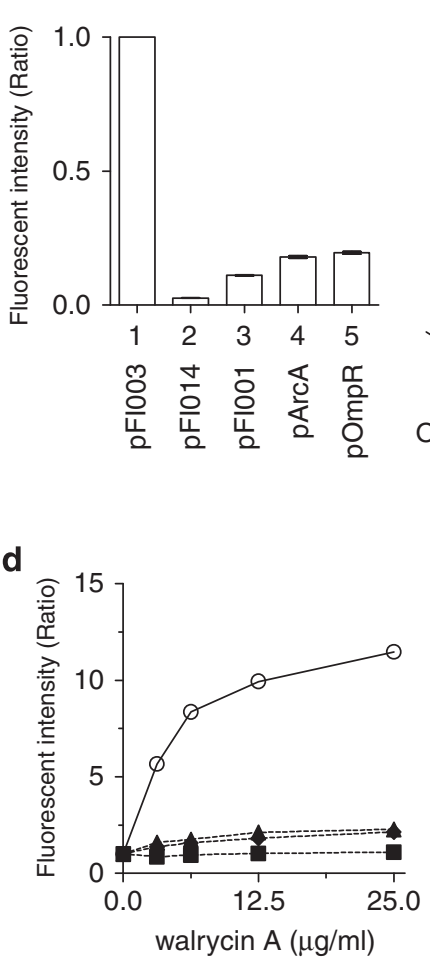

N100 HD (WalR or RRs)
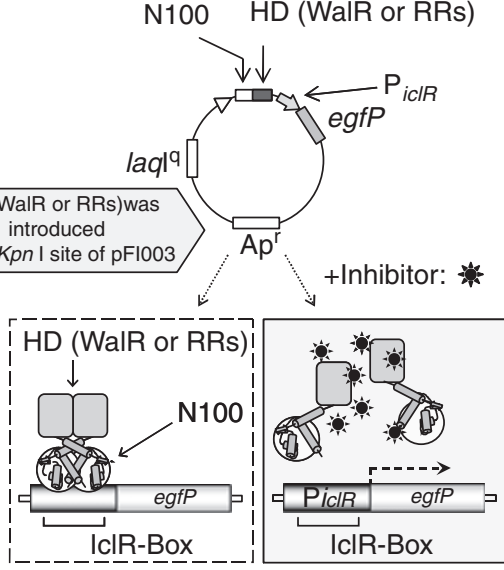

Repression of EGFP
C<smiles>COc1ccc(O)c2ccccc12</smiles><smiles>Cn1nc(-c2ccc(C(F)(F)F)cc2)nc2c(=O)n(C)c(=O)nc1-2</smiles>

walrycin B

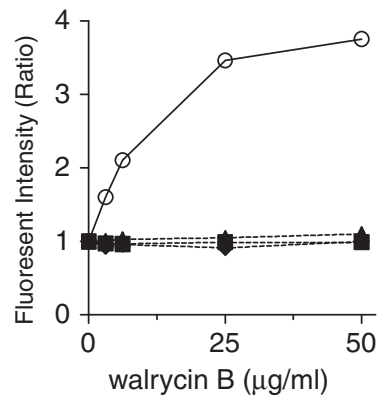

Figure 2 Screening of WalR inhibitors. (a) High-throughput screening system. N100, N-terminal 100-a.a. DNA-binding domain of IcIR; HD, homodimerization domain; ICIR Box, -21 to +14 on iclR promoter region; RRs, response regulators (WaIR, ArcA and OmpR). (b) Repression of EGFP intensity by response regulators and IcIR. JM109 containing pFI003 (column 1), pFI014 (column 2), pFI001 (column 3), pArcA (column 4) or pOmpR (column 5) was cultured and fluorescence intensity was measured as described in Materials and methods, indicated as a value relative to that of JM109 containing pFI003. (c) Structural formulas of walrycins A and B. (d) Induction of EGFP by walrycins A or B. JM109 containing pFI014 (open circles), pFI001 (squares), pArcA (triangles) or pOmpR (diamonds) was cultured in the presence or absence of walrycins $A$ or B. Fluorescence intensity is presented here as a value relative to that in the absence of walrycins $\mathrm{A}$ or $\mathrm{B}$. 
Table 1 Bacterial strains and plasmids used in this study

\begin{tabular}{|c|c|c|}
\hline Strain or plasmid & Relevant genotype or description & Source or reference \\
\hline \multicolumn{3}{|l|}{ E. coli } \\
\hline BL21(DE3) & $\mathrm{F}^{-}$, ompT, $h s d S_{\mathrm{B}}\left(\mathrm{r}_{\mathrm{B}}^{-} \mathrm{m}_{\mathrm{B}}^{-}\right), g a l, d c m$ (DE3) & Laboratory stock \\
\hline \multicolumn{3}{|l|}{ B. subtilis } \\
\hline 168 & $\operatorname{trpc2}$ & Laboratory stock \\
\hline \multicolumn{3}{|l|}{ Plasmid } \\
\hline pFI001 & 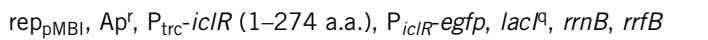 & Furuta et al. ${ }^{20}$ \\
\hline pFIOO3 & 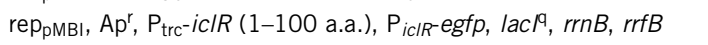 & Furuta et al. ${ }^{20}$ \\
\hline pFIO14 & pFI003, trc- $^{-i c / R}$ (1-100 a.a.)-walR (B. subtilis) & This work \\
\hline pArcA & pFI003, $\mathrm{P}_{\text {trc }}-i c / R(1-100$ а.а.)-arcA (E. coli) & This work \\
\hline pETBsFF & pET21a(+), Nde I-Xho I fragment encoded B. subtilis WaIR & This work \\
\hline pSCSawalRKH & $\mathrm{pSC}-\mathrm{B}, \mathrm{S}$. aureus walRKyycH & This work \\
\hline pETSa315VR & pET101/D-TOPO, S. aureus walR (vicR) & This work \\
\hline pETBsG-tru & pET21a(+), Bam HI-Not I fragment encoded B. subtilis WalKtru & This work \\
\hline pAN18 & plasmid vector & Fukushima et al. ${ }^{21}$ \\
\hline $\mathrm{pABF}$ & pAN18, B. subtilis walR & This work \\
\hline $\mathrm{pMK}_{4} \mathrm{P}_{\text {prot }}$ & plasmid vector & Archambaud et al. ${ }^{22}$ \\
\hline pSD1-7 & $\mathrm{pMK}_{4} \mathrm{P}_{\text {prot }}$, S. aureus walR & This work \\
\hline
\end{tabular}

$X b a \mathrm{I}$ and then inserted into $K p n \mathrm{I}$ and $\mathrm{Xba \textrm {I }}$ sites of pAN $18^{21}$ to obtain pABF. A DNA fragment containing the $S$. aureus walR was amplified by PCR using primers OSA211 and OSA250 and S. aureus chromosomal DNA as a template. The PCR product was digested with BamHI and PstI and then cloned into $\mathrm{pMK}_{4} \mathrm{P}_{\text {prot }}{ }^{22}$ to obtain $\mathrm{pSD1-7}$. For WalR protein purification, B. subtilis walR was amplified by PCR with pBY33 as the template and primers BsF-A-F and BsF-B-R. The PCR product was digested with NdeI and XhoI and then inserted into XhoI and NdeI sites of pET21a(+) (Novagen, Madison, WI, USA). The constructed plasmid was named pETBsFF. For WalKtru (C-terminal region, 204-611 a.a.) protein purification, B. subtilis walK was amplified by PCR with pBY33 as the template and primers YYCG(TRU)-F and YYCG(TRU)-R. The PCR product was digested with BamHI and NotI and then inserted into BamHI and NotI sites of pET21a(+). The constructed plasmid was named pETBsG-tru. A DNA fragment containing walRKyycH of $S$. aureus was amplified by PCR with $S$. aureus $\mathrm{N} 315^{23}$ genomic DNA as the template and primers SAvicop-up and SAvicop-2dw. The PCR product was directly ligated to PSC-B (Stratagene, La Jolla, CA, USA) and was named pSCSawalRKH. S. aureus walR was subcloned from pSCSawalRKH to pET101/D-TOPO (Invitrogen, Carlsbad, CA, USA) by using primers TP-Sa315VR-F and TP-Sa315VR-3 and named pETSa315VR.

\section{Chemical library}

Two different kinds of chemical libraries were used for screening: one was produced by a new bioconversion system in recombinant $E$. coli cells; ${ }^{24}$ the other contained 10000 compounds from ChemBridge Corporation (San Diego, CA, USA).

\section{Screening method}

A chemical library was screened using the E. coli-based high-throughput enhanced green fluorescent protein (EGFP) screening system. JM109 cells containing plasmids pFI001, pFI003, pFI014, pArcA or pOmpR were grown at $37^{\circ} \mathrm{C}$ in $\mathrm{LB}$ (Luria-Bertani) medium containing ampicillin. Then, $1 \mu \mathrm{l}$ of this culture was added to 96 -titer wells containing $200 \mu \mathrm{l}$ of LB-ampicillin medium and $1 \mu \mathrm{l}$ of drugs, and the cultures were grown at $37^{\circ} \mathrm{C}$ for $20 \mathrm{~h}$. Fluorescence was measured using a Wallac 1420 ARVOsx (PerkinElmer Life Sciences, Boston, MA, USA) with excitation and emission wavelengths of 485 and $535 \mathrm{~nm}$, respectively, and the optical density at $595 \mathrm{~nm}\left(\mathrm{OD}_{595}\right)$ was measured using a Model 3550 Microplate Reader (Bio-Rad Laboratories, Hercules, CA, USA). For normalization the fluorescence intensity of each sample was divided by its $\mathrm{OD}_{595}$ value.

\section{Drug susceptibility assay}

The MICs were examined by the serial agar dilution method using MuellerHinton agar (Difco Laboratories, Detroit, MI, USA). ${ }^{25}$ The MIC was determined as the lowest concentration at which growth was significantly inhibited.

\section{Trans-phosphorylation of WalR by phosphorylated WalKtru}

To prepare phosphorylated (P-) WalKtru, WalKtru $(0.5 \mu \mathrm{M})$ was incubated at room temperature for $15 \mathrm{~min}$ in kinase buffer (50 mM Tris- $\mathrm{HCl} \mathrm{pH} \mathrm{8.5,200} \mathrm{mM}$ $\mathrm{KCl}, 100 \mathrm{~mm} \mathrm{NH} \mathrm{N}_{4} \mathrm{Cl}, 25 \mathrm{~mm} \mathrm{MgCl}, 12.5 \mathrm{~mm}$ ATP and $\left.0.4 \mu \mathrm{Ci}\left[\gamma_{-}{ }^{32} \mathrm{P}\right] \mathrm{ATP}\right)$. After WalR $(1 \mu \mathrm{M})$ was incubated at room temperature for $60 \mathrm{~min}$ in the presence of walrycins A or B, a P-WalKtru mixture was added and incubated for $15 \mathrm{~min}$. The reaction was terminated by adding SDS sample buffer. The samples were analyzed using SDS-polyacrylamide gel electrophoresis. The gel was dried and exposed on an image plate and analyzed using FLA-7000 (Fuji Film, Tokyo, Japan).

\section{RNA preparation and complementary DNA synthesis}

For quantitative real-time PCRs (qRT-PCRs), the B. subtilis 168 and S. aureus N315 strains were grown with aeration to an OD 600 of 0.4 , after which walrycins A or B was added and they were cultivated for another $5 \mathrm{~min}$. The cells were harvested by centrifugation and immediately suspended in RNA-later (Ambion, Austin, TX, USA). RNA preparations were then performed with an SV total RNA Isolation System (Promega, Madison, WI, USA), followed by a 
DNase I treatment with the TURBO DNA-free Kit (Ambion) to eliminate residual contamination by genomic DNA. Reverse transcription reactions were performed with the High Capacity complementary DNA Reverse Transcription Kit (ABI, Foster City, CA, USA).

\section{qRT-PCRs}

Primers (Supplementary Table S2) were selected with Primer Express Software Version 3.0 (ABI). qRT-PCRs were performed with complementary DNA $(0.1 \mathrm{ng}), 250 \mathrm{nM}$ gene-specific primer pair and PowerSYBR Green Master Mix (ABI). PCR amplification, detection and analyses were performed using StepOne Real Time PCR system (ABI). PCR conditions included an initial denaturation step at $95^{\circ} \mathrm{C}$ for $10 \mathrm{~min}$, followed by a 40 -cycle amplification ( $95^{\circ} \mathrm{C}$ for $15 \mathrm{~s}$ and $60^{\circ} \mathrm{C}$ for $60 \mathrm{~s}$ ). The specificity of the amplified product and the absence of primer dimer formation were verified by generating a melting curve. Melting curve analysis was performed by an initial denaturation step of $95^{\circ} \mathrm{C}$ for $15 \mathrm{~s}$ followed by $60^{\circ} \mathrm{C}$ for $1 \mathrm{~min}$ and again $95^{\circ} \mathrm{C}$ for $15 \mathrm{~s}$. Fluorescence was measured continually during the melting curve cycle (a stepwise $0.3^{\circ} \mathrm{C}$ temperature), beginning at $60^{\circ} \mathrm{C}$. The absence of contaminating genomic DNA was verified by testing each sample in control reactions without a previous reverse transcription step. The critical threshold cycle $\left(C_{\mathrm{T}}\right)$ was defined for each sample.

The expression levels were normalized using the 16S rRNA gene as an internal standard whose transcriptional level did not vary under our experimental conditions. Each assay was performed in triplicate and repeated using at least three independent RNA samples.

\section{Preparation of ${ }^{32} \mathrm{P}$-end-labeled DNA probes}

Reverse primer (10 pmol, 5'-AAGACGTCATTGATAAAGACGCACTCCGGT-3') was phosphorylated with $10 \mu \mathrm{Ci}\left[\gamma^{3}{ }^{32} \mathrm{P}\right]$ ATP $\left(5000 \mathrm{Ci} \mathrm{mmol} 1^{-1}\right.$, PerkinElmer) by using T4 polynucleotide kinase (Takara Bio, Otsu, Japan). The promoter fragment $\left(\mathrm{P}_{\text {yocH }}\right)$ was amplified by PCR using B. subtilis 168 genomic DNA as template, Ex Taq DNA polymerase (Takara Bio), and ${ }^{32}$ P-labeled reverse primer and forward primer (5'-AATACAGGGCTTATGCAAGGATGACAGACTA-3'). The labeled fragments were purified and the radioactivity was measured with a liquid scintillation counter.

\section{Gel mobility shift assay}

Gel mobility shift assays were carried out as described in Yamamoto et al. ${ }^{26}$ The ${ }^{32} \mathrm{P}$-labeled probe was incubated at $30{ }^{\circ} \mathrm{C}$ for $10 \mathrm{~min}$ with purified $\mathrm{His}_{6}$-protein in binding buffer. After addition of a DNA dye solution $\left(40 \%\left(\mathrm{vv}^{-1}\right)\right.$ glycerol, $0.025 \%\left(\mathrm{wv}^{-1}\right)$ bromophenol blue and $0.025 \%\left(\mathrm{wv}^{-1}\right)$ xylene cyanol, the mixtures were subjected to $6 \%$ polyacrylamide gel electrophoresis.

\section{Fluorescent dyes and fluorescence microscopy}

Exponentially growing cells were mounted on 2\% agarose pads containing growth medium for microscopy use and stained with Synapto Green (membrane) and SYBR green I (DNA). Images $(\times 1000)$ were acquired on a Radiance 2100 confocal laser scanning microscope (Bio-Rad Laboratories).

\section{Protein purification}

E. coli BL21(DE3) cells containing the pETBsFF, pETSa315VR, pKH55-21 or pKH43-21 plasmids ${ }^{27}$ were grown in $2 \times$ YT medium at $30{ }^{\circ} \mathrm{C}$. When the cell density reached an $\mathrm{OD}_{600}$ of 0.6 , IPTG was added at the final concentration of $1 \mathrm{~mm}$, and the cell suspension was cultivated for another $3 \mathrm{~h}$ before the cells were harvested by centrifugation, washed with lysis buffer $(50 \mathrm{~mm}$ Tris- $\mathrm{HCl}$ $(\mathrm{pH} 8.0), 100 \mathrm{~mm} \mathrm{NaCl})$ and then stored at $-80^{\circ} \mathrm{C}$ until use. Frozen cells were suspended in lysis buffer containing $1 \mathrm{~mm}$ phenylmethylsulphonyl fluoride and then lysed by sonication. The lysates were cleared by centrifugation and filtered through a $0.22-\mu \mathrm{m}$ filter. The filtrate was loaded onto a HisTrap HP column (ÄKTA prime system; GE Healthcare Bio-Science, Piscataway, NJ, USA) equilibrated with the lysis buffer, and $\mathrm{His}_{6}$-protein was eluted using an imidazole gradient from 0 to $500 \mathrm{~mm}$. The fractions with the target protein were dialyzed against storage buffer (10 mM Tris- $\mathrm{HCl}(\mathrm{pH} 8.0), 0.1 \mathrm{~mm}$ EDTA, $0.1 \mathrm{~mm}$ dithiothreitol and $10 \%\left(\mathrm{vv}^{-1}\right)$ glycerol $)$, loaded onto a HiTrap Q FF (GE Healthcare Bio-Science) and eluted with a $\mathrm{NaCl}$ gradient of $0-500 \mathrm{~mm}$ in storage buffer. Fractions containing the $\mathrm{His}_{6}$-tagged proteins were collected and loaded onto a HiTrap Desalting column (GE Healthcare Bio-Science).

\section{Analytical size-exclusion chromatography}

Size-exclusion chromatography analysis was performed on a fast-performance liquid chromatography ÄKTA system using a Superdex 200 10/300 GL or Superdex 75 10/300 GL (all three devices from GE Healthcare Bio-Science). Experiments were carried out at $4{ }^{\circ} \mathrm{C}$ and a flow rate of $0.25 \mathrm{ml} \mathrm{min}^{-1}$ using $10 \mathrm{~mm}$ Tris- $\mathrm{HCl}$ ( $\mathrm{pH} 8.0$ ), $150 \mathrm{~mm} \mathrm{NaCl}, 0.1 \mathrm{~mm}$ EDTA and $0.1 \mathrm{~mm}$ dithiothreitol as the mobile phase while monitoring absorbance at $280 \mathrm{~nm}$ was determined. Samples $(300 \mu \mathrm{l})$ were prepared by incubating $2.5 \mathrm{nmol}$ of protein in $10 \mathrm{~mm}$ Tris- $\mathrm{HCl}(\mathrm{pH} 8.0), 0.1 \mathrm{~mm}$ EDTA and $0.1 \mathrm{~mm}$ dithiothreitol at $30^{\circ} \mathrm{C}$ for $60 \mathrm{~min}$ in the presence or absence of $25 \mu \mathrm{g} \mathrm{ml}^{-1}$ of walrycin A. Before injection, samples were filtered through $0.22-\mu \mathrm{m}$ filters.

\section{Limited proteolysis with trypsin}

After WalR proteins were digested with trypsin (Promega) as previously described, ${ }^{28}$ they were separated by SDS-polyacrylamide gel electrophoresis, transferred to a polyvinylidine difluoride membrane (Immobilon-P transfer membranes; Millipore, Bedford, MA, USA), and detected using anti-WalR antibody and enhanced chemiluminescence plus western blotting system (GE Healthcare Bio-Science).

\section{RESULTS AND DISCUSSION}

High-throughput screening of inhibitors targeting WalR

From the results of the crystal structure analysis of the Thermotoga maritime IclR protein, ${ }^{29}$ it was predicted that this protein is composed of two domains: the N-terminal DNA-binding domain containing the winged helix-turn-helix motif and a regulatory domain in the Cterminal of the protein, which is involved in its binding to a signal molecule. These two domains are linked by an $\alpha$-helix. ${ }^{29}$ The binding of a signal molecule to the $\mathrm{C}$-terminal domain of IclR is presumed to repress the transcription of target genes by modulating either DNAbinding activity or multimerization of IclR. ${ }^{29}$ Database homology searches have identified many IclR-like proteins, known as the IclR family, which show a conserved C-terminal region. E. coli IclR also forms a dimer and regulates both the aceBAK operon and its own expression. ${ }^{30-32}$ Furuta et al. ${ }^{20}$ previously reported that the region between 100 and 274 a.a. of IclR is involved in dimerization and that this region can be replaced with other functional homodimerization domains (Figure 1). To design a high-throughput screening system targeting the WalR RR, we constructed a chimeric repressor gene consisting of sequences encoding the 100 amino-terminal amino acid residues of IclR that constitute the DNA-binding domain (N100) fused to the walR, $\operatorname{arcA}$ or $\operatorname{ompR} R \mathrm{RR}$ genes (Figure 2a). To quantify the dimerization capacity of the resulting chimerical repressor proteins in terms of fluorescence intensity, the egfP reporter gene was placed directly downstream from the $i c l R$ promoter (Figure $2 a$ ) as described previously. ${ }^{20}$ In the presence of inhibitors targeting the RRs, the egfP gene is expressed and EGFP fluorescence intensity increases. WalR, ArcA and OmpR belong to the OmpR/PhoB subfamily, in which dimerization is essential for DNA-binding and transcriptional activation. Consequently, they are all capable of suppressing expression from the iclR promoter (Figure $2 \mathrm{~b}$ ). These results suggest that this highthroughput system could be used to screen inhibitors of RRs containing dimerization domains. To identify selective WalR inhibitors by measuring EGFP fluorescence intensity, we screened a chemical library against E. coli strains JM109/pFI014 (WalR) and JM109/pFI001 (IclR) in 96-titer wells. Through this screening process, we identified two compounds, compound A (4-methoxy-1-naphthol; CAS no. 84-85-5) and compound B (1,6-dimethyl-3-[4-(trifluoromethyl)phenyl]pyrimido[5,4-e][1,2,4] triazine-5,7-dione; CAS no. 878419-78-4) 
(Figure 2c) as WalR inhibitors. Both compounds strongly increased fluorescence intensity in a dose-dependent manner in strain JM109/ pFI014 but did not significantly change fluorescence intensity of strain
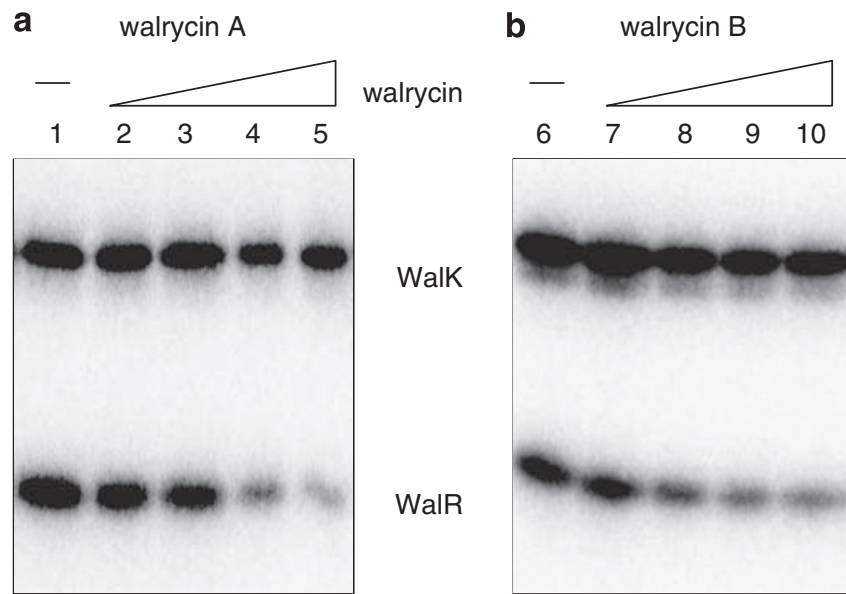

WalK

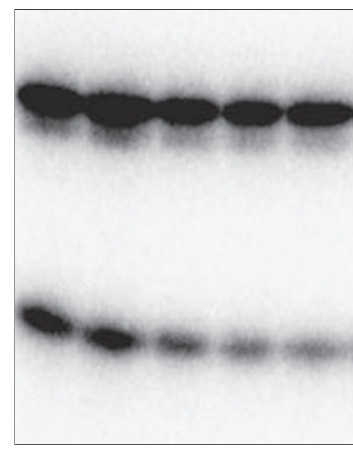

Figure 3 Inhibition of phosphotransfer from P-WalKtru to WalR. Phosphotransfer from P-WalKtru to WalR was performed in the presence or absence of walrycin A (a, lanes 1-5) or walrycin B (b, lanes 6-10): lane 1, $0 \mu \mathrm{m}$; lane 2, 22.3 $\mu \mathrm{m}$; lane 3, 44.8 $\mu \mathrm{m}$; lane 4, 89.6 $\mu \mathrm{m}$; lane 5, 179.2; lane 6, $0 \mu \mathrm{m}$; lane 7, 3.6 $\mu \mathrm{m}$; lane 8, 14.5 $\mu \mathrm{m}$; lane 9, 58.1 $\mu \mathrm{m}$; lane 10, 232.4 $\mu \mathrm{m}$.
JM109/pFI001 (Figure 2d). Furthermore, to clarify that they actually target WalR, we analyzed their effect on phosphotransfer from autophosphorylated WalKtru (cytoplasmic HK region) to WalR (Figure 3). As a result, phosphotransfer from P-WalKtru to WalR was inhibited with increased concentrations of compounds A and B. These results strongly suggest that both compounds target WalR to inactivate the IclR-WalR chimera repressor for inducing GFP expression. Therefore, we named them walrycin A and walrycin B.

\section{Regulation of WalR regulon genes}

To establish that these compounds exert an effect specifically on the WalK/WalR system, the effects of walrycins on in vivo expression of WalR regulon genes ${ }^{4,5,8,33,34}$ were analyzed using qRT-PCR). In B. subtilis, WalR activates expression of $y d j M$ (cell wall-associated protein), yocH (cell wall hydrolase) and $y v c E$ ( $c w l O$, cell wall endopeptidase), whereas it negatively regulates expression of yoeB (modulator of autolysin activity) and yjeA (peptideglycan deacetylase). In $S$. aureus, WalR activates expression of several cell wall metabolism genes including isa $A$ (cell wall transglycosylase) and $s s a A$ (cell wall amidase). As shown in Figure 4, addition of walrycins A and B to B. subtilis led to lowered expression of $y d j M, y o c H$ and $y v c E$ and increased expression of yoeB and yjeA within $5 \mathrm{~min}$ (Figures $4 \mathrm{a}$ and b). Similarly, transcription of $S$. aureus WalR regulon genes isa $A$ and $s s a A$ stopped within 5 min of adding walrycins $A$ and $B$ to the culture (Figures $4 \mathrm{a}$ and b). Furthermore, both walrycin compounds had antimicrobial a

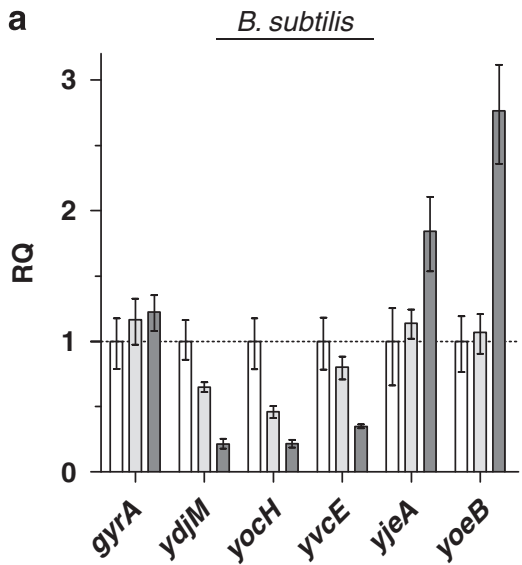

b

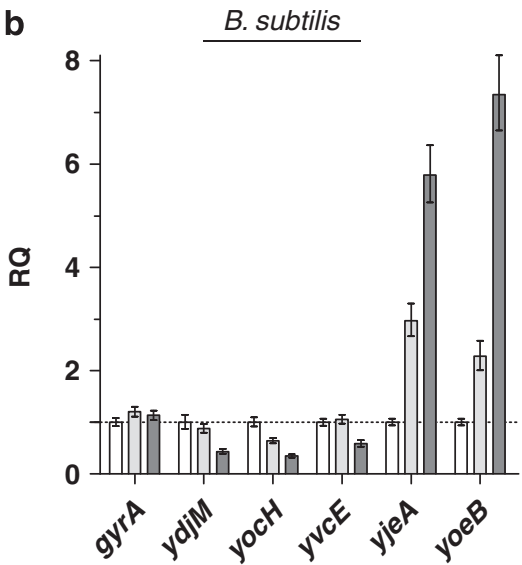

$\underset{\Upsilon}{\text { O }}$
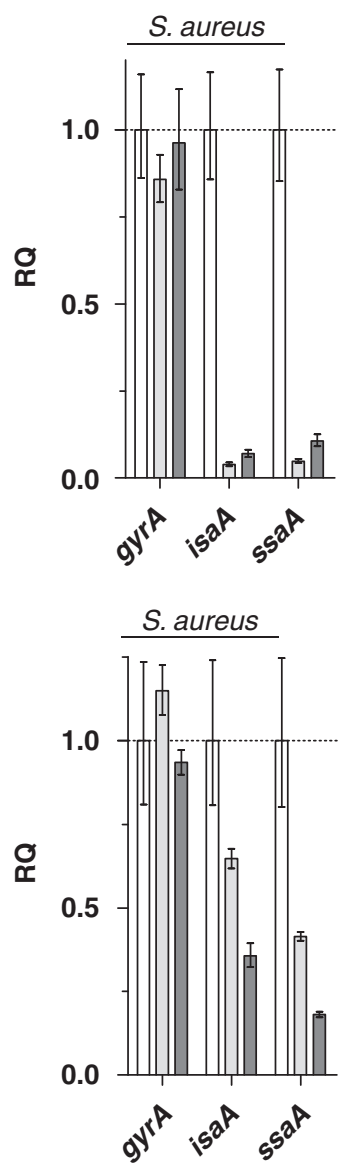

Figure 4 Transcriptional regulation of WalR regulon genes by walrycins $A$ or B. Total RNAs were extracted from $B$. subtilis or $S$.aureus cells, after which walrycin A (a; 0,25 and $50 \mu \mathrm{g} \mathrm{ml}^{-1}$ for B. subtilis and 0,50 and $100 \mu \mathrm{g} \mathrm{m}^{-1}$ for $S$. aureus) or walrycin $\mathrm{B}\left(\mathbf{b} ; 0,0.78\right.$ and $1.56 \mu \mathrm{g} \mathrm{m} \mathrm{l}^{-1}$ for $B$. subtilis and 0 , 3.13 and $6.25 \mu \mathrm{g} \mathrm{ml}^{-1}$ for $S$. aureus) was added, and they were cultivated for $5 \mathrm{~min}$. After reverse transcription, specific complementary (c)DNAs were quantified using qRT-PCR. The results are expressed as the means of relative quantification to that of $0 \mu \mathrm{ml}^{-1}$ and s.d. of triplicate experiments using primers specific for WalR regulon genes, gyrA and 16S rRNA (normalizing gene). 
a

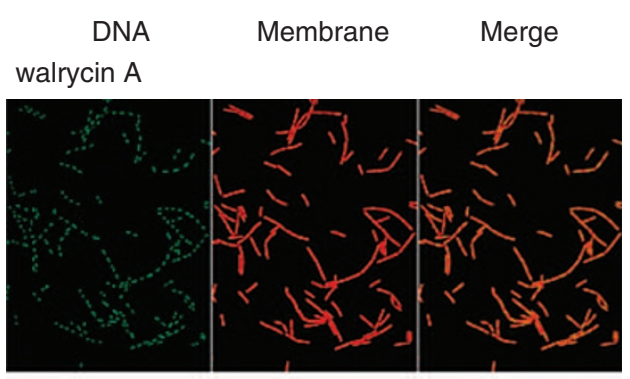

walrycin B

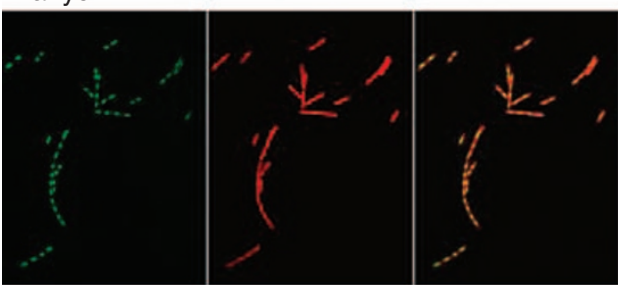

Control

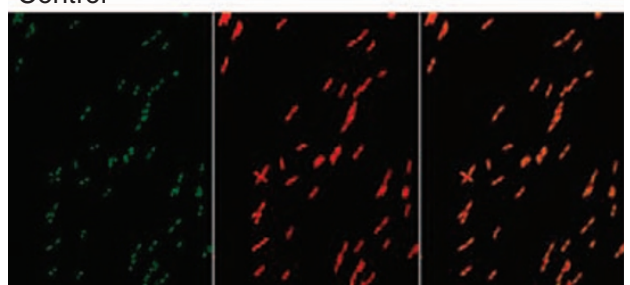

b

DNA Membrane Merge

walrycin A

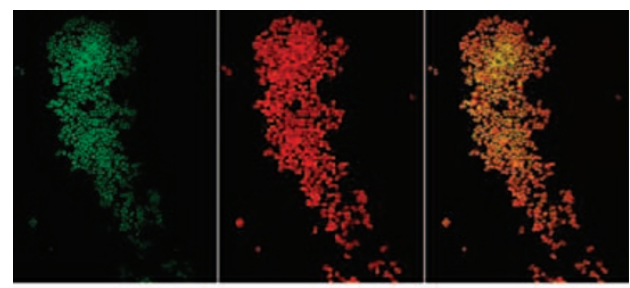

walrycinB

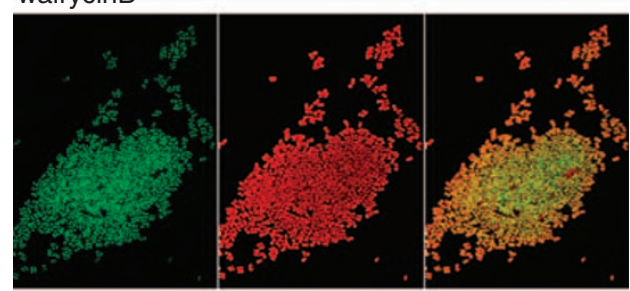

Control

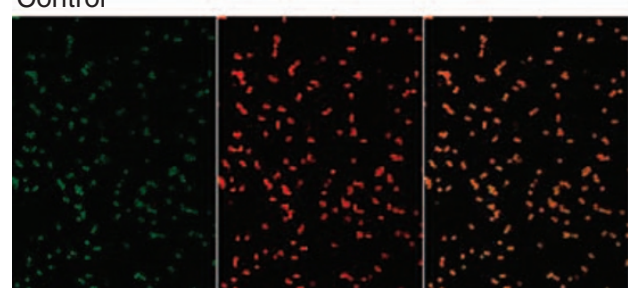

Figure 5 Morphology changes induced by walrycins A or B. Confocal laser scanning microscopy of exponentially growing cells of $B$. subtilis 168 (a) and

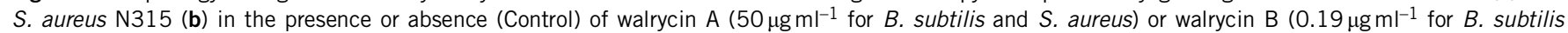
and $0.78 \mu \mathrm{g} \mathrm{ml}^{-1}$ for $S$. aureus).

activity, with MICs of walrycins A and B of 64 and $0.39 \mu \mathrm{g} \mathrm{ml}^{-1}$ for B. subtilis 168 and 128 and $3.13 \mu \mathrm{g} \mathrm{ml}^{-1}$ for $S$. aureus $\mathrm{N} 315$, respectively. These results indicate that walrycins A and B target WalR and lead to cell death in both B. subtilis and S. aureus.

The MICs of walrycin B against B. subtilis and S. aureus were shown to be stronger than those of walrycin $A$, at which concentration the WalR regulon gene expressions were specifically effected to cause cell death. These results suggest that walrycins A and B actually target WalR in the B. subtilis and S. aureus. On the other hand, walrycins A and B inhibited phosphotransfer from WalK to WalR at similar concentrations in vitro. However, walrycin A induced EGFP at lower concentrations than did walrycin B in a high-throughput genetic system using E. coli (Figure 2). Walrycin A might enter E. coli cells easily, but not B. subtilis and S. aureus, whereas the reverse is true for walrycin B.

To confirm that walrycins target the WalR RR, the effects of WalR overproduction were analyzed. When WalR was overexpressed from a multi-copy plasmid (B. subtilis strain 168/pABF and S. aureus strain $\mathrm{N} 315 / \mathrm{pSD} 1-7)$, the MICs for walrycin A were increased twice over those of the control strains $\left(168 / \mathrm{pAN} 18\right.$ and $\left.\mathrm{N} 315 / \mathrm{pMK}_{4} \mathrm{P}_{\text {prot }}\right)$ to 128 and $256 \mu \mathrm{g} \mathrm{ml}^{-1}$, respectively. Under the same conditions, MICs for walrycin B were increased fourfold for B. subtilis and twofold for $S$. aureus. These results indicate that the walrycins specifically target WalR to cause cell death in both B. subtilis and S. aureus.

In this study we have shown that walrycins inhibit WalR activity in B. subtilis and S. aureus. These activities are involved in many crucial cell functions and influence cell development. Because autolysins are involved in the separation of daughter cells, one would expect WalR- depleted cells to be filamentous or aggregated. We used a confocal laser microscope to examine the effect of walrycin A on the morphology of B. subtilis and S. aureus cells. As shown in Figure 5, untreated B. subtilis and $S$. aureus cells had a typically short rod morphology or were well separated, whereas walrycins A and B-treated cells formed extremely long aseptate filaments or aggregates. Furthermore, after B. subtilis was incubated in the presence of walrycins A or B, cell lysis was observed (data not shown). These results are consistent with the previously reported phenotypes of WalR-depleted mutants. ${ }^{4,6,33}$

\section{A mode of action}

B. subtilis and S. aureus WalRs, which were overproduced and purified from E. coli, revealed both monomeric and dimeric forms (Figure 6a-1, 2), whereas ArcA and OmpR formed the monomer as previously reported. ${ }^{35-37}$ After WalR was treated with walrycin A at $30^{\circ} \mathrm{C}$ for $60 \mathrm{~min}$ followed by size-exclusion chromatography, a decrease in the concentration of WalR monomer and a concomitant increase in the dimeric form (Figure 6a-1, 2) were observed. This increase in the proportion of dimeric protein was not observed when purified ArcA or OmpR was incubated with walrycin A (Figure 6a-3, 4). As these results suggest that walrycin A can exert an effect specifically on monomeric WalR to increase dimer formation, we performed a sizeexclusion chromatography on fractionated WalR-monomers with or without walrycin A treatment. As shown in Figure 6a-5, almost half of the monomer had transitioned into the dimeric form. When dimeric WalR was treated with walrycin A under the same conditions, however, no change was observed (Figure 6a-6). These results clearly show that walrycin A exerts an effect specifically on the monomeric 

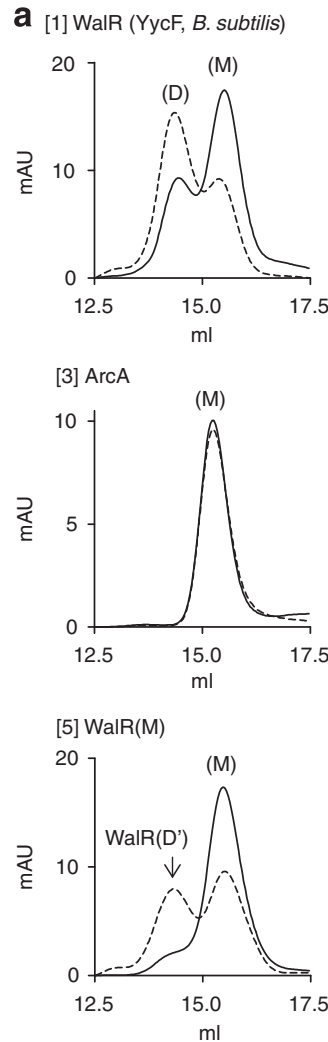

[2] WalR (VicR,S. aureus)

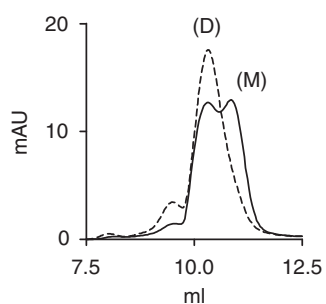

[4] OmpR

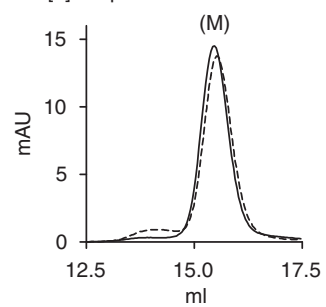

[6] WalR(D)

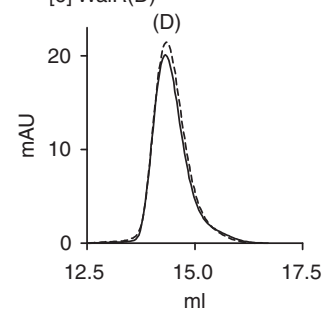

b $\operatorname{WalR}(\mathrm{D}) \quad \operatorname{WaIR}\left(\mathrm{D}^{\prime}\right)$

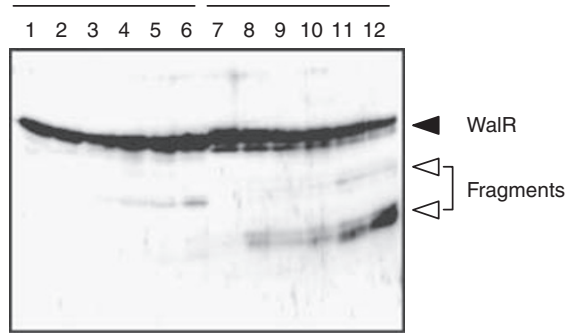

c
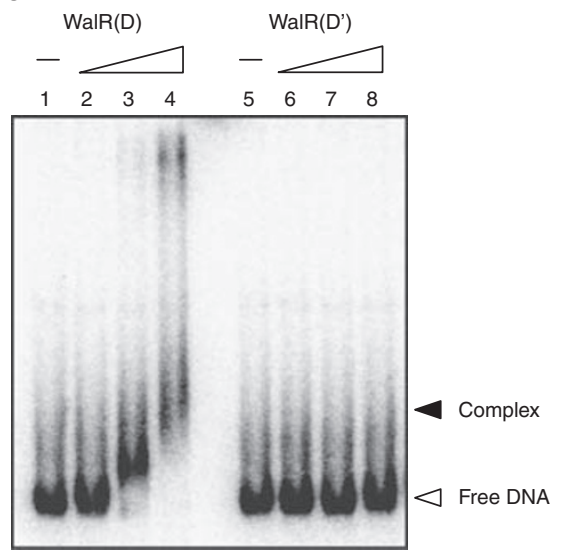

Figure 6 A mode of action of walrycin A targeting WalR. (a) Walrycin A interacts with monomeric WalR. After each protein (1-6) was incubated at $30{ }^{\circ} \mathrm{C}$ for $60 \mathrm{~min}$ without (solid line) or with (dotted line) $25 \mu \mathrm{g} \mathrm{ml}^{-1}$ of walrycin A, size-exclusion chromatography was performed as described in Materials and methods. WalRs (B. subtilis), ArcA and OmpR were separated using a Superdex 200 10/300 GL column, and S. aureus WalR was separated using a Superdex 75 10/300 GL column (GE Healthcare Bio-Science). 1, WalR (B. subtilis); 2, WalR (S. aureus); 3, ArcA (E. coll); 4, OmpR (E. coll); 5, the monomeric WaIR (B. subtilis); 6, dimeric WaIR (B. subtilis). M, monomer; D, dimer. (b) Conformational changes of WalR(D) and WalR(D'). WalR was digested with trypsin (protein to trypsin, 20:1). A total of $30 \mathrm{ng}$ of WalR(D) (lanes 1-6) and WalR(D') (lanes 7-12) were digested with $1.5 \mathrm{ng}$ of trypsin for 0 (lanes 1 and 7), 15 (lanes 2 and 8), 30 (lanes 3 and 9), 60 (lanes 4 and 10), 90 (lanes 5 and 11) and 120 min (lanes 6 and 12). (c) Gel mobility shift analysis with WalR(D) and WaIR( $\left.D^{\prime}\right)$. A radiolabeled DNA fragment carrying the promoter of yocH was prepared as described in Materials and methods. The mobility of fragments without WalR addition is shown in lanes 1 and 5. Lanes 2-4 (WalR(D)) and 6-8 (WalR(D')) show mobility after binding of 2.5 (lanes 2 and 6), 5 (lanes 3 and 7) or 10 pmol (lanes 4 and 8) of WalR.

form of WalR to increase the formation of WalR dimers. We designated the dimer that formed after the reaction of walrycin A and the WalR monomer as WalR $\left(\mathrm{D}^{\prime}\right)$ and evaluated its structural characteristics (Figure 6b). WalR(D) and WalR( $\left(D^{\prime}\right)$ were digested with trypsin, and the fragments were separated by SDS-polyacrylamide gel electrophoresis and analyzed using western blotting (Figure 6b). Results showed fragments arising from trypsin-digested $\operatorname{WalR}\left(\mathrm{D}^{\prime}\right)$, which were not detected in the WalR(D)-digested sample. These results indicate a possible altered conformation for WalR $\left(\mathrm{D}^{\prime}\right)$ generated by the reaction with walrycin A when compared with endogenous WalR(D). The ability of WalR $\left(\mathrm{D}^{\prime}\right)$ to bind to the promoter region of the B. subtilis yocH gene, which contains a WalR-binding site, was analyzed using gel mobility shift analysis (Figure 6c). Results showed that WalR(D) bound to the WalR box of $\mathrm{P}_{\text {yoch }}$ and formed a DNAWalR(D) complex that migrated at a slower rate than the free DNA. However, no protein/DNA complex was formed when the yocH probe was incubated with WalR $\left(\mathrm{D}^{\prime}\right)$, indicating that no binding took place. These results show that walrycin A reacts with the WalR monomer to form a WalR dimer (WalR $\left(\mathrm{D}^{\prime}\right)$ ) with a different conformation from that of the endogenous dimeric WalR, and which lacks the ability to bind to WalR(D) operator sites (Figure 7).

Concerning the difference in the mode of action between walrycins $\mathrm{A}$ and $\mathrm{B}$, we conducted an in vitro phosphotransfer experiment from
Extracellular compartment

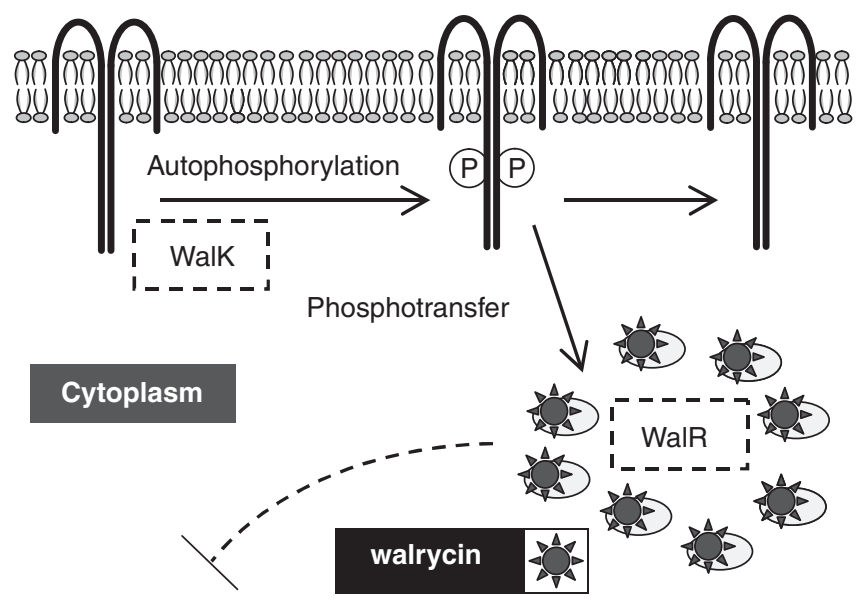

000000000001 walR regulon genes

Figure $7 \mathrm{~A}$ model of walrycin action. 
P-WalKtru to WalR in the presence or absence of walrycins (Figure 3). As a result, phosphotransfer was inhibited by both walrycins A and B. These results suggest that they likely inhibit WalR activity by a similar mechanism: by targeting WalR. Walrycin B is known as an analog of toxoflavin (a phytotoxin from Burkholderia glumae), which has been shown to have a strong MIC for B. subtilis and S. aureus but whose mode of action is not clear. ${ }^{38}$ The compound could also interact with WalR to cause bactericidal effects. Recently, Kim et al. ${ }^{39}$ reported that toxoflavin binds ToxR (an LysR-type transcriptional regulator) to activate synthetic gene expression of toxoflavin.

Walrycins are a new class of potent small-molecule compounds that kill bacterial cells by targeting the RR WalR and inhibiting this essential signal transduction pathway. They not only have therapeutic potential but will also prove to be useful reagents for the further study of the WalK/WalR TCS.

\section{ACKNOWLEDGEMENTS}

We thank Yamamoto K for plasmids pKH55-21 and pKH43-21 and Yamamoto K, Kobayashi K, Nagamatsu T and Nishiyama S for valuable discussions. We also thank Eguchi $\mathrm{Y}$ and Ho $\mathrm{K}$ for critically reviewing the manuscript and Kubo N, Shiraki S, Suzuki T, Otsuka A and Hirai Y for technical support. This work was supported by a Grant-in-Aid for Scientific Research (A, 20248012) of the Japan Society for the Promotion of Science (JSPS), the Research and Development Program for New Bio-Industry Initiatives (2006-2010) of the Bio-Oriented Technology Research Advancement Institution (BRAIN). Work in the group of Msadek T was supported by research funds from the European Commission (Grants BACELL Health LSHG-CT-2004-503468, StaphDynamics LHSM-CT-2006-019064 and BaSysBio LSHG-CT-2006-037469), the Centre National de la Recherche Scientifique (CNRS URA 2172), and the Institut Pasteur (GPH no. 9).

1 Utsumi, R. (ed). (2008) Bacterial Signal Transduction: Networks and Drug Targets (Springer, New York, NY).

2 Dubrac, S., Bisicchia, P., Devine, K. M. \& Msadek, T. A matter of life and death: cell wall homeostasis and the WalKR (YycGF) essential signal transduction pathway. Mol. Microbiol. 70, 1307-1322 (2008).

3 Gotoh, Y. et al. Two-component signal transduction as potential drug targets in pathogenic bacteria. Curr. Opin. Microbiol. (in press).

4 Dubrac, S., Boneca, I. G., Poupel, O. \& Msadek, T. New insights into the WalK/WaIR (YycG/YycF) essential signal transduction pathway reveal a major role in controlling cell wall metabolism and biofilm formation in Staphylococcus aureus. J. Bacteriol. 189, 8257-8269 (2007).

5 Bisicchia, P. et al. The essential YycFG two-component system controls cell wall metabolism in Bacillus subtilis. Mol. Microbiol. 65, 180-200 (2007).

6 Fabret, C. \& Hoch, J. A. A two-component signal transduction system essential for growth of Bacillus subtilis: implications for anti-infective therapy. J. Bacteriol. 180, 6375-6383 (1998).

7 Martin, P. K., Li, T., Sun, D., Biek, D. P. \& Schmid, M. B. Role in cell permeability of an essential two-component system in Staphylococcus aureus. J. Bacteriol. 181, 3666-3673 (1999).

8 Dubrac, S. \& Msadek, T. Identification of genes controlled by the essential YycG/YycF two-component system of Staphylococcus aureus. J. Bacteriol. 186, 1175-1181 (2004).

9 Hilliard, J. J., Goldschmidt, R. M., Licata, L., Baum, E. Z. \& Bush, K. Multiple mechanisms of action for inhibitors of histidine protein kinases from bacterial twocomponent systems. Antimicrob. Agents Chemother. 43, 1693-1699 (1999).

10 Yamamoto, K. et al. Antibacterial agents that inhibit histidine protein kinase YycG of Bacillus subtilis. Biosci. Biotechnol. Biochem. 65, 2306-2310 (2001).

11 Watanabe, T. et al. Isolation and characterization of inhibitors of the essential histidine kinase, YycG in Bacillus subtilis and Staphylococcus aureus. J. Antibiot. 56, 1045-1052 (2003).

12 Watanabe, T., Okada, A., Gotoh, Y. \& Utsumi, R. Inhibitors targeting two-component signal transduction. Adv. Exp. Med. Biol. 631, 229-236 (2008).
13 Qin, Z. et al. Structure-based discovery of inhibitors of the YycG histidine kinase: new chemical leads to combat Staphylococcus epidermidis infections. BMC Microbiol. 6, 96 (2006).

14 Mizuno, T. \& Tanaka, I. Structure of the DNA-binding domain of the OmpR family of response regulators. Mol. Microbiol. 24, 665-667 (1997).

15 Okajima, T. et al. Response regulator YycF essential for bacterial growth: X-ray crystal structure of the DNA-binding domain and its PhoB-like DNA recognition motif. FEBS Lett. 582, 3434-3438 (2008).

16 Gao, R., Mack, T. R. \& Stock, A. M. Bacterial response regulators: versatile regulatory strategies from common domains. Trends Biochem. Sci. 32, 225-234 (2007).

17 Howell, A., Dubrac, S., Noone, D., Varughese, K. I. \& Devine, K. Interactions between the YycFG and PhoPR two-component systems in Bacillus subtilis: the PhoR kinase phosphorylates the non-cognate YycF response regulator upon phosphate limitation. Mol. Microbiol. 59, 1199-1215 (2006).

18 Gueriri, I., Bay, S., Dubrac, S., Cyncynatus, C. \& Msadek, T. The Pta-AckA pathway controlling acetyl phosphate levels and the phosphorylation state of the DegU orphan response regulator both play a role in regulating Listeria monocytogenes motility and chemotaxis. Mol. Microbiol. 70, 1342-1357 (2008).

19 Stephenson, K. \& Hoch, J. A. Developing inhibitors to selectively target two-component and phosphorelay signal transduction systems of pathogenic microorganisms. Curr. Med. Chem. 11, 765-773 (2004).

20 Furuta, E. et al. Targeting protein homodimerization: a novel drug discovery system. FEBS Lett. 579, 2065-2070 (2005).

21 Fukushima, S., Itaya, M., Kato, H., Ogasawara, N. \& Yoshikawa, H. Reassessment of the in vivo functions of DNA polymerase I and RNase $\mathrm{H}$ in bacterial cell growth. J. Bacteriol. 189, 8575-8583 (2007).

22 Archambaud, C., Gouin, E., Pizarro-Cerda, J., Cossart, P. \& Dussurget, O. Translation elongation factor EF-Tu is a target for Stp, a serine-threonine phosphatase involved in virulence of Listeria monocytogenes. Mol. Microbiol. 56, 383-396 (2005).

23 Kuroda, M. et al. Whole genome sequencing of methicillin-resistant Staphylococcus aureus. Lancet 357, 1225-1240 (2001).

24 Misawa, N. et al. Synthesis of vicinal diols from various arenes with a heterocyclic, amino or carboxyl group by using recombinant Escherichia coli cells expressing evolved biphenyl dioxygenase and dihydrodiol dehydrogenase genes. Tetrahedron 61, 195-204 (2005).

25 Igarashi, M. et al. Pargamicin A, a novel cyclic peptide antibiotic from Amycolatopsis sp. J. Antibiot. 61, 387-393 (2008).

26 Yamamoto, K., Ogasawara, H., Fujita, N., Utsumi, R. \& Ishihama, A. Novel mode of transcription regulation of divergently overlapping promoters by PhoP, the regulator of two-component system sensing external magnesium availability. Mol. Microbiol. 45, 423-438 (2002).

27 Yamamoto, K. et al. Functional characterization in vitro of all two-component signal transduction systems from Escherichia coli. J. Biol. Chem. 280, 1448-1456 (2005).

28 Kenny, L. J., Bauer, M. D. \& Silhavy, T. J. Phosphorylation-dependent conformational changes in OmpR, an osomoregulatory DNA-binding protein of Escherichia coli. Proc. Natl Acad. Sci. USA 92, 8866-8870 (1995).

29 Zhang, R. G. et al. Crystal structure of Thermotoga maritima 0065, a member of the IcIR transcriptional factor family. J. Biol. Chem. 277, 19183-19190 (2002).

30 Yamamoto, K. \& Ishihama, A. Two different modes of transcription repression of the Escherichia coli acetate operon by IcIR. Mol. Microbiol. 47, 183-194 (2003).

31 Donald, L. J. et al. Mass spectrometric study of the Escherichia coli repressor proteins, Ic1R and Gc1R, and their complexes with DNA. Protein Sci. 10, 1370-1380 (2001).

32 Gui, L., Sunnarborg, A., Pan, B. \& LaPorte, D. C. Autoregulation of $i c / R$, the gene encoding the repressor of the glyoxylate bypass operon. J. Bacteriol. 178, 321-324 (1996).

33 Fukuchi, K. et al. The essential two-component regulatory system encoded by yyc $F$ and yycG modulates expression of the ftsAZ operon in Bacillus subtilis. Microbiology 146, 1573-1583 (2000).

34 Howell, A. et al. Genes controlled by the essential YycG/YycF two-component system of Bacillus subtilis revealed through a novel hybrid regulator approach. Mol. Microbiol. 49, 1639-1655 (2003).

35 Kenney, L. J. Structure/function relationships in $\mathrm{OmpR}$ and other winged-helix transcription factors. Curr. Opin. Microbiol. 5, 135-141 (2002).

36 Toro-Roman, A., Mack, T. R. \& Stock, A. M. Structural analysis and solution studies of the activated regulatory domain of the response regulator ArcA: a symmetric dimer mediated by the $\alpha 4-\beta 5-\alpha 5$ face. J. Mol. Biol. 349, 11-26 (2005).

37 Rhee, J. E. et al. Amino acids important for DNA recognition by the response regulator OmpR. J. Biol. Chem. 283, 8664-8677 (2008).

38 Nagamatsu, T. et al. Syntheses of 3-substituted 1-methyl-6-phenylpyrimido[5,4-e]1,2,4-triazine-5,7(1 H,6H)-diones (6-phenyl analogs of toxoflavin) and their 4-oxides, and evaluation of antimicrobial activity of toxoflavins and their analogs. Chem. Pharm. Bull. 41, 362-368 (1993).

$39 \mathrm{Kim}$, J. et al. Biochemical evidence for ToxR and ToxJ binding to the tox operons of Burkholderia glumae and mutational analysis of ToxR. J. Bacteriol. 191, 4870-4878 (2009).

Supplementary Information accompanies the paper on The Journal of Antibiotics website (http://www.nature.com/ja) 